\title{
A Cooperative Model to Improve Hospital Equipments and Drugs Management
}

\author{
Ilaria Baffo ${ }^{1,2}$, Giuseppe Confessore ${ }^{1}$, Giacomo Liotta ${ }^{1}$, and Giuseppe Stecca ${ }^{1}$ \\ ${ }^{1}$ Istituto di Tecnologie Industriali e Automazione, Consiglio Nazionale delle Ricerche, \\ Area della Ricerca Roma 1, Strada della neve, Via Salaria Km 29,300, \\ 00010 Montelibretti, Rome, Italy \\ ${ }^{2}$ Dipartimento di Ingegneria dell'Impresa, Università di Roma "Tor Vergata", \\ Via del Politecnico 1, 00133, Rome, Italy \\ \{Ilaria.Baffo, Giuseppe. Confessore, Giacomo. Liotta, \\ Giuseppe.Stecca\}@itia.cnr.it
}

\begin{abstract}
The cost of services provided by public and private healthcare systems is nowadays becoming critical. This work tackles the criticalities of hospital equipments and drugs management by emphasizing its implications on the whole healthcare system efficiency. The work presents a multi-agent based model for decisional cooperation in order to address the problem of integration of departments, wards and personnel for improving equipments, and drugs management. The proposed model faces the challenge of $(i)$ gaining the benefits deriving from successful collaborative models already used in industrial systems and (ii) transferring the most appropriate industrial management practices to healthcare systems.
\end{abstract}

Keywords: Multi Agent Systems, Healthcare Management Systems, Logistics.

\section{Introduction}

The cost of services provided by public and private healthcare systems is nowadays becoming critical. Since in several countries, hospital revenues are not more cost based, reduction in costs are becoming the critical point for the sustainability of all medical structures. Moreover, the great size of the hospital, while on one hand allows to exploit the economies of scale, on the other hand makes the internal dynamics of a hospital a complex non-linear structure [1] that is difficult to manage.

This work tackles the issues of hospital equipments, personnel and drugs management by emphasizing its implications on the whole healthcare system efficiency. The adopted approach is based on the assumption that the hospital is a complex system composed by a great number of entities and processes. Camarinha-Matos and Afsarmanesh [2] defined a collaborative network as a network consisting of autonomous, distributed, heterogeneous entities that collaborate to better achieve common or compatible goals. Following this definition, the application of a Multi Agent System (MAS) to collaborative network modeling turns out to be natural.

The MASs have been widely exploited in several application fields in order to govern the complexity through cooperation and decentralization of decisions when 
competitive requests and divergent objectives have to considered. In this work a MAS-based approach is adopted in order to offer a model able to manage the complexity of a healthcare system while:

- fostering technological innovations through information sharing and recovery;

- fostering organizational innovations for more efficient and effective distributed decisions whenever possible.

A MAS can be herein considered as the basic methodological factor for then deploying technological enablers (e.g., ICT) and managerial enablers (e.g., a cooperative decision system of the healthcare organization). The system is supposed to consist of several decisional and operational agents that can be related to departments or wards. Every agent is considered as a single decisional entity that influences the achievement of system's goals. The work presents a model of agents' cooperation for addressing the problem of departments, wards and personnel integration in order to improve the efficiency regarding drugs, equipments, and personnel management. The overall goal of the system is related to the need of providing, with a fixed budget and consequently a fixed set of resources, the highest level of customers served with high service level. In particular, the cooperative model for drugs management is based on cooperative actions for obtaining service effectiveness while reducing inventory costs through information sharing and coordination. According to Camarinha-Matos and Afsarmanesh [3] more than one modeling perspective must be integrated in order to properly design a Collaborative Networked Organization (CNO). We will describe architectural aspects presenting the agents and their roles in the $\mathrm{CNO}$, component aspects describing technological aspects in terms of hardware/software needed in the $\mathrm{CNO}$ in order to fulfill the overall objective, functional aspects describing peculiar processes of the CNO.

The paper is organized as follows: Section 2 presents the main issues concerning healthcare management systems with respect to the management of equipments and drugs. The problem is described in Section 3. In Section 4 the agent-based cooperative model is presented and discussed. Conclusions follow.

\section{Resource Management in Healthcare Systems}

The efficiency of healthcare systems is influenced by increased demand for quality, technology investments and increased drug supplies [4]. The consequent trade-offs among effectiveness, efficiency and equity objectives have fostered the development of theory and applications concerning health economics and management science [4].

Since in several countries, hospital revenues are not any more cost based, cost reductions are becoming the critical point for the sustainability of all medical structures. For this reason some managers tried to apply some principles and techniques deriving from management science, for improving the efficiency and effectiveness of hospital facilities. In order to obtain cost savings, hospitals need to review their activities, to identify the costs associated with the activities and reduce them, to classify the activities in terms of added-value, and to decrease or eliminate not added-value activities [5]. By applying this methodology, called Activity Based Management (ABM), a lot of organizations focused their efforts to improve activities belonging to the logistic 
department. This department is a vital part of a hospital because it may have responsibilities for activities like purchasing, receiving, inventory management, management information systems, telemedicine, transportation, and home care services. Although these activities do not represent the core mission for the hospital, they take part into definition of service level offered by the organization. Consequently, it is important to examine the activities of this department to improve services and possibly reduce costs while adopting lean service processes. The internal dynamics of a hospital represents a complex non-linear structure [1]. Planning and management of hospital daily operations require a thorough understanding of the system with information for decision making [1].

Literature review confirms that several studies address management issues although many gaps need to be bridged, for instance with respect to integrated logistics of healthcare systems. De Angelis et al. [6] investigate the problem of assigning resources and servers (e.g., doctors, beds, instruments) to services. Akcali et al. [7] tackle the problem of optimizing hospital bed capacity planning through a network flow approach. Harper [1] proposes an integrated simulation tool (PROMPT, Patient and Resource Operational Management Planning Tool) for the planning and management of hospital resources such as beds, operating theatres, and needs for nurses, doctors and anaesthetists. Van Merode et al. [8] study the potential adoption of Enterprise Resource Planning (ERP) systems in hospitals while facing the issues of planning and control processes and determining ERP systems requirements. Liu et al. [9] and De Treville et al. [10] argue that efficiency and quality of healthcare management have been enhanced by the computerization of healthcare information. Several studies concern the information management, workflow (see [11]) and automation in healthcare systems. Thornett [12] discusses potential roles, introduction benefits and difficulties related to computer decision support systems within the practice in primary healthcare. It can be concluded that organizational innovations and enabling ICTbased solutions are essential conditions to reach the efficiency and effectiveness improvement in hospital operations management.

\section{Problem Description}

Thanks to the effort of an Italian research project's partners it was possible to analyze and synthesize the main problems related to drugs and equipment management within some Italian hospital structures. In particular, the study of the AS-IS processes and the survey on the employers showed several problems in the following processes: $(i)$ central drugs management, (ii) drugs management in each ward, (iii) medical equipment management.

With regard to the first aspect, the problems arise from some lacks in the continuous monitoring of the central drugstore. When the responsible of the drugs is not present, the nurses, filling a paper register, are free to use the pharmacy. That approach could produce an inappropriate management of the drugs or errors in the related information records. Other problems come from the variability of the drugs in terms of codes and packaging. When a drug order is fulfilled, it is possible that the required drug it is not available. Consequently, it is substituted with an equivalent drug. It was observed that equivalent drugs are registered with different codes. This 
approach generates difficulties in the drugstore management. Regarding the drugs management in each ward, instead, the followings problems can describe the scenario analyzed by researchers, medical personnel and industrial managers:

- The stock levels of the wards drugstores can be out of control whether not recorded in the computer system of the hospital.

- The drug order fulfillment of the central drugstore could not properly take into account the stock levels of wards drugstores. Since information on the availability of medicines in the wards drugstores could not be recorded into the computer system, the nurses may need to check the availability by physical inspection in the wards.

- The decisions about the fulfilling of drugs are made by the nurses. Minimum supply concept is not used in the fulfilling process. The orders are often made without information about the stock availability. That approach can generate an over abundance of drugs. Furthermore, it was observed that the nurses tend to make large orders in order to increase their responsiveness capability.

- Problems arise in the management of medicinal products expiration dates with possible unreasonable costs of not usable drugs.

- With regard to the movements of medicinal products between the wards drugstores, it can happen that the nurses of a ward instead of replenishing the local drugstore by using the central pharmacy of the hospital, take the medicinal products from other wards drugstores. This procedure can imply that the nurses do not physically move throughout proper the hospital structure and consequently the drugs that have been taken could not be registered. It was observed that the movements of medicinal products can tend to generate conflicts between nurses of different wards.

Other problems coming from medical equipments management are:

- High cost of the management of hospital equipments. It has to be considered that the weekly movement of the required materials take 1.5 workdays of the store employee.

- The hospital equipments store is not managed by a computer system. The stock level is not exactly known. In order to find the equipment necessary for a patient it is often necessary for a nurse to move physically into the store where there is not a standard procedure to find the selected equipment but it needs to be founded by visual inspection.

- The localization of the equipments into the hospital structure is not defined. When something is not found in the equipments store, the nurse needs to check this stuff in all the hospital building.

- Tags are used to assign each instrument to each patient. The tag can be lost or it deteriorates too much, making it ineffective with the risk of loosing data of the patients.

Currently, several internal possible solutions are object of studies to improve the quality of the drug management. It seems that the most efficient manner to organize the system is to create a centralized unit, represented by the central drugstore, with higher control on the whole drugs and equipments management process. 


\section{Methodology: The Cooperative Model}

This section proposes a model based on multi-agent theory in order to formalize the cooperative model under two dimensions: $(i)$ organizational innovation for cooperative process and (ii) technological framework supporting the cooperative process. Moreover, as Camarinha-Matos and Afsarmanesh [3] claim, the collaborative network requires the development of models, not only as a help to better understand the area, but also as the basis for the development of methods and tools for better decisionmaking [3]. The realized model will be illustrated through the description of the agents, the explanation of the collaborative decisional process and the technological architecture. Finally, a discussion on ongoing and foreseen applications is made.

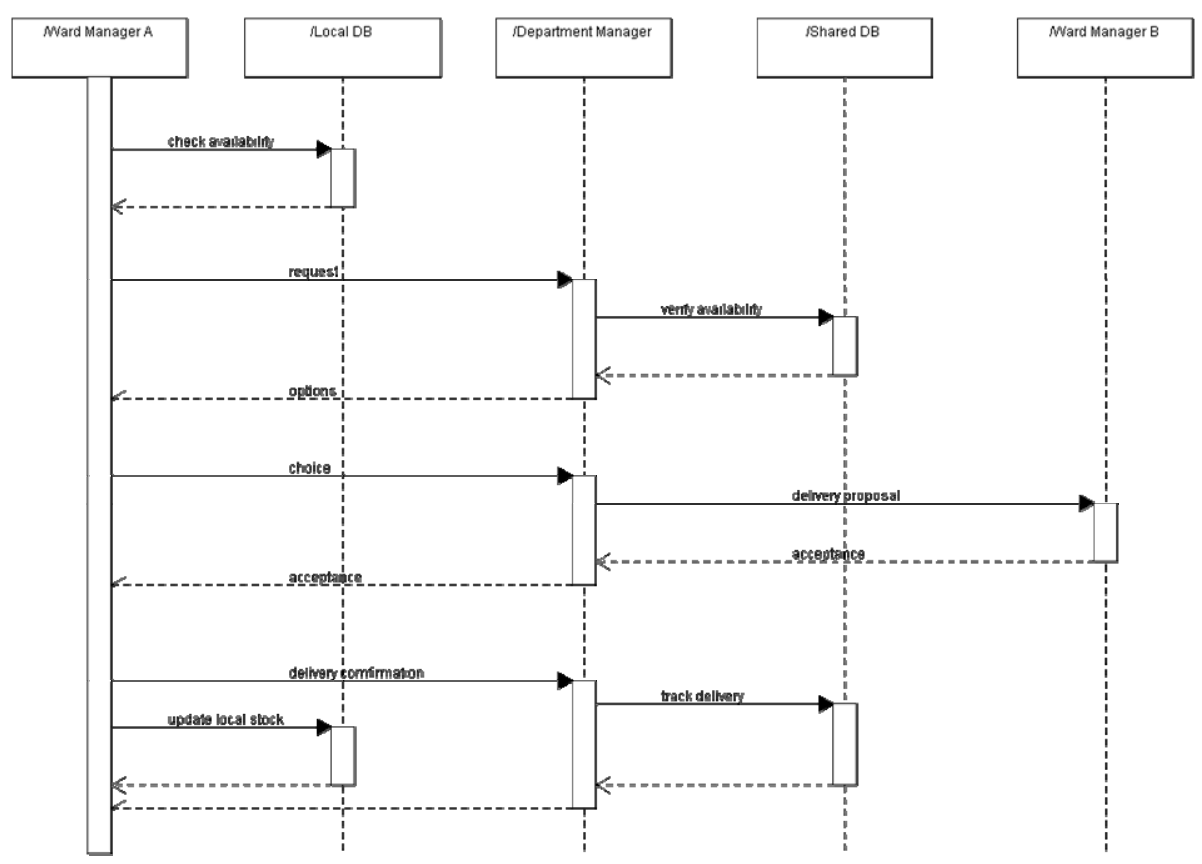

Fig. 1. UML sequence diagram for a general request

\subsection{Description of Agents}

The reengineered system should enable the collaboration among medical wards in the processes of material procurement and resource allocations. As described in Section 3 , the lack of integrated information systems and the not formalized collaboration rules are the main obstacles to the development of a collaborative process. A model composed by a central manager and by medical ward managers is proposed. They act as interfaces of the central warehouse and the local ward warehouses. The Department Manager (DM) is a coordinator of resource allocation, and of material management processes. The Ward Managers (WM) represents the local decision maker for 
the ward and department objectives. The DM is a composition of three specialized decision makers: (i) Equipment manager, (ii) Drugs procurement manager, (iii) $\mathrm{Hu}$ man resource manager.

The DM is an enabler for centralized information and an enabler to collaboration among wards for drugs procurement, equipment allocation and human resource assignment.

\subsection{Collaborative Decisional Process among Agents}

The decisional process is general and foresees an interaction between WMs and DM each time that a request is generated by the WM.

As showed in Figure 1, a WM sends a procurement request to the DM. The DM verifies the central and the local warehouses. Then, it sends a set of procurement options to the WM who replies with its choice. If the choice is an inter-ward delivery (for instance from WM B), then this request is forwarded to the ward chosen. The process terminates with a delivery from WM B to WM A and a notification of the delivery to the DM.

\subsection{Applications and Discussion}

The architecture shown in Figure 2 supports the collaborative process and the information centralization in a hospital system. The information systems must be fully integrated in order to track deliveries and material consumption.

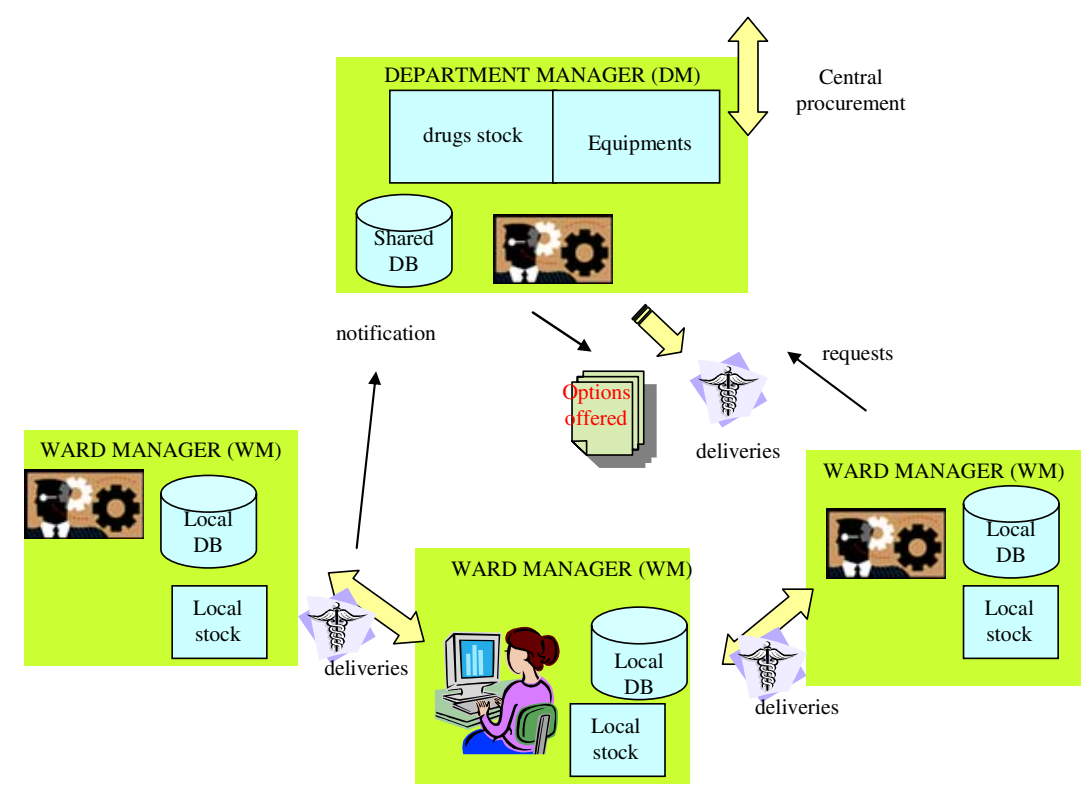

Fig. 2. System Architecture 
Very promising technological enablers for effectively managing, even in real time, particular products or systems (equipment, medical devices, drugs) are the Radio Frequency Identification (RFId) technologies. The introduction in healthcare structures of a pervasive technology such as the RFId with complementary decision tools likely entails the redesign of key logistic processes as described in this work. On the other hand the presence of RFId technologies raises the complexity of the system. In fact, together with the introduction of RFId different critical issues must be resolved or managed such as privacy management and signal transmission interferences. The tracking points for deliveries and material consumption are detailed in the collaborative procurement procedures. In order to enable physical tracking and localization, RFId technology can be used in combination with automatic delivery devices. Automatic delivery devices impose the authentication and enable the tracking of the delivery. Moreover, introducing the prescription of the doctors in the hospital information system, it is possible to perform a crosscheck between the prescribed drugs and the drug quantities that have been taken from the stores. Overall, three main areas of application of the system have been identified: (i) drugs procurement, (ii) equipment management, (iii) human resource scheduling. The application to drugs procurement allows the improvement of the FIFO material management strategy and effective use of decentralized warehouses. When the requests arrive to DM, the drugs manager verifies the stocks and propose different delivery options based on the expiry dates, distance from the warehouses, lead-time of procurement from suppliers.

If an inter-ward delivery is chosen then it must be tracked. The ward from which the drug has been sourced will be supplied once the supplier procurement will arrive. The application to the equipment management foresees the localization and the tracking of the equipments uses. This information enables the correct allocation of the equipments. The utilization requests should allow to plan maintenance and substitution for assuring a high service level in term of equipment' s availability . The application to Human resource scheduling foresees the use of a medical skills database for planning the deployment of medical teams. The Human resource manager should verify that every operation of medical personnel is tracked in terms of duration and used skills.

Through the adoption of the herein presented MAS-based approach for modelling the drugs and equipments management processes, it is expected in particular a better medicinal products inventory management while introducing process innovations and enabling technologies. The logistic department should then have a strategic role inside of a hospital due to the visibility on state and location of drugs, equipments, personnel, patients, etc.

\section{Conclusion and Acknowledgments}

In this paper a collaborative model for efficiency improvements in healthcare systems has been proposed. The proposed model faces the challenges of $(i)$ gaining the benefits deriving from successful collaborative models already used in industrial systems and (ii) transferring the most appropriate industrial management practices to healthcare systems. The proposed approach could allow a better medicinal products inventory management. The drug expiration problems can be avoided and the costs significantly 
reduced. To develop the solution proposed in this paper the authors propose a MASbased approach to model the drugs and equipments management processes. With this solution they propose an innovation of the processes based on re-design of operations and on the introduction of technological solutions. The logistic department acquires a strategic role inside of hospital because it can become the gathering point of all the hospital information related to state and location of drugs, equipments, personnel, patients, etc. Future research steps foresee an improvement of the model while possibly including performance indicators (e.g., time, costs and service level) in the analysis.

This work is partially supported by the Italian region "Lombardia" within the "Lean Healthcare" project that is in progress. The presented work is based on part of the project's outcomes. The project joins industrial managers and researchers with the aim to transfer the most known industrial management practices to healthcare systems. The authors would like to acknowledge the whole project's partnership, the hospital structures that have allowed the analysis of their internal processes, and the financing authority.

\section{References}

1. Harper, P.R.: A Framework for Operational Modelling of Hospital Resources. Health Care Manage. Sci. 5, 165-173 (2002)

2. Camarinha-Matos, L.M., Afsarmanesh, H.: Collaborative Networks: A New Scientific Discipline. J. Intell. Manuf. 16, 439-452 (2005)

3. Camarinha-Matos, L.M., Afsarmanesh, H.: A Comprehensive Modeling Framework for Collaborative Networked Organizations. J. Intell. Manuf. 18, 529-542 (2007)

4. Athanassopoulos, A., Gounaris, C.: Assessing the Technical and Allocative Efficiency of Hospital Operations in Greece and its Resource Allocation Implications. Eur. J. Oper. Res. 133, 416-431 (2001)

5. Aptel, O., Pourjalali, H.: Improving Activities and Decreasing Costs of Logistics in Hospitals. A Comparison of U.S. and French Hospitals. The International Journal of Accounting 36, 65-90 (2001)

6. De Angelis, V., Felici, G., Impelluso, P.: Integrating Simulation and Optimisation in Health Care Centre Management. Eur. J. Oper. Res. 150, 101-114 (2003)

7. Akcali, E., Coté, M.J., Lin, C.: A Network Flow Approach to Optimizing Hospital Bed Capacity Decisions. Health Care Manage. Sci. 9, 391-404 (2006)

8. Van Merode, G.G., Groothuis, S., Hasman, A.: Enterprise Resource Planning for Hospitals. Int. J. Med. Inform. 73, 493-501 (2004)

9. Liu, D.R., Wu, I.C., Hsieh, S.T.: Integrating SET and EDI for Secure Healthcare Commerce. Comp. Stand. Inter. 23, 367-381 (2001)

10. De Treville, S., Smith, I., Rolli, A., Arnold, V.: Applying Operations Management Logic and Tools to Save Lives: A Case Study of The World Health Organization's Global Drug Facility. J. Oper. Manag. 24, 397-406 (2006)

11. Dang, J., Hedayati, A., Hampel, K., Toklu, C.: An Ontological Knowledge Framework for Adaptive Medical Workflow. Journal of Biomedical Informatics 41, 829-836 (2008)

12. Thornett, A.M.: Computer Decision Support Systems in General Practice. Int. J. Inform. Manage. 21, 39-47 (2001) 